Abstracts for ASA Fall Meeting - 28November-2December 2016 - Honolulu, HI

Jeremy Kenny and Clothilde Giacomoni

\title{
Comparison of Spatial Correlation Parameters between Full and Model Scale Launch
} Vehicles

The current vibro-acoustic analysis tools require specific spatial correlation parameters as input to define the liftoff acoustic environment experienced by the launch vehicle. Until recently these parameters have not been very well defined. A comprehensive set of spatial correlation data were obtained during a scale model acoustic test conducted in 2014. From these spatial correlation data, several parameters were calculated: the decay coefficient, the diffuse to propagating ratio, and the angle of incidence. Spatial correlation data were also collected on the EFT-1 flight of the Delta IV vehicle which launched on December $5^{\text {th }}, 2014$. A comparison of the spatial correlation parameters from full scale and model scale data will be presented. 